\title{
Obesidade abdominal em adultos: Prevalência e fatores associados
}

\author{
Abdominal obesity in adults: Prevalence and associated factors \\ Obesidad abdominal en adultos: Prevalencia y factores asociados
}

Thaís Sasaki

ORCID: https://orcid.org/0000-0003-2351-9086 Universidade Estadual do Paraná, Brasil E-mail: thais.sasaki118@gmail.com

Heloá Costa Borim Christinelli

ORCID: https://orcid.org/0000-0003-0772-4194

Universidade Estadual do Paraná, Brasil E-mail: heloa.borim@hotmail.com

Kely Paviani Stevanato

ORCID: https://orcid.org/0000-0003-1872-8246 Universidade Estadual do Paraná, Brasil E-mail: kelystevanato@gmail.com

Elen Ferraz Teston

ORCID: https://orcid.org/0000-0001-6835-0574 Universidade Federal de Mato Grosso do Sul, Brasil E-mail: elen-1208@hotmail.com

Vinícius Luís da Silva

ORCID: https://orcid.org/0000-0001-6228-8124 Universidade Estadual do Paraná, Brasil E-mail: vinicius.luissilva@hotmail.com Maria Antonia Ramos Costa

ORCID: https://orcid.org/0000-0001-6906-5396 Universidade Estadual do Paraná, Brasil E-mail: maria.costa@unespar.edu.br

Carlos Alexandre Molena Fernandes

ORCID: https://orcid.org/0000-0002-4019-8379 Universidade Estadual do Paraná, Brasil E-mail: carlosmolena126@gmail.com

\begin{abstract}
Resumo
A obesidade abdominal está associada a um aumento exponencial no risco de morbimortalidade, visto que o padrão de distribuição de gordura corporal é o fator de risco mais significativo em processos mórbidos do que a obesidade generalizada. Estimar a prevalência de obesidade abdominal e identificar os fatores associados. Estudo piloto, descritivo transversal, com abordagem quantitativa. A amostra por conveniência foi constituída por 50 indivíduos com idade entre 18 a 50 anos. Inicialmente foi realizada avaliação antropométrica dos participantes e posteriormente a aplicação de um questionário para definir o perfil e hábitos da população com circunferência da cintura alterada $(\geq 88 \mathrm{~cm}$ para mulheres ou $\geq 102 \mathrm{~cm}$ para homens). Os dados foram analisados por meio de estatística descritiva e regressão linear utilizandose o software Epi Info ${ }^{\mathrm{TM}}$. Dentre os participantes $58 \%(\mathrm{n}=29)$ apresentaram obesidade abdominal. As variáveis que apresentaram significância ( $\mathrm{p}$-valor $\leq 0.05$ ) com a obesidade abdominal foram: ser casado, não ser da raça branca, ter 8 anos ou mais de escolaridade; ter IMC indicativo de sobrepeso e obesidade generalizada e não consumir bebida alcóolica. A obesidade abdominal bem como a obesidade generalizada, constitui-se como um grande desafio a ser trabalhado pelos profissionais da saúde, considerando que uma fração significativa da população se encontra nessa circunstância. Nessa perspectiva, a prevenção e o controle da obesidade abdominal devem prover ações que auxiliem os indivíduos na adoção de estilo de vida saudável.
\end{abstract}

Palavras-chave: Obesidade; Peso corporal; Estilo de vida, Doença crônica; Fatores de risco.

\begin{abstract}
Abdominal obesity is associated with an exponential increase in the risk of morbidity and mortality, since the pattern of body fat distribution is the most significant risk factor in morbid processes than generalized obesity. Estimate the prevalence of abdominal obesity and identify the associated factors. Pilot, descriptive cross-sectional study with a quantitative approach. The convenience sample consisted of 50 individuals aged 18 to 50 years. Initially, an anthropometric assessment of the participants was carried out and then a questionnaire was applied to define the profile and habits of the population with altered waist circumference ( $\geq 88 \mathrm{~cm}$ for women or $\geq 102 \mathrm{~cm}$ for men). The data were analyzed using descriptive statistics and linear regression using the Epi Info ${ }^{\mathrm{TM}}$ software. Among the participants, 58\% $(\mathrm{n}=29)$ had abdominal obesity. The variables that showed significance ( $\mathrm{p}$-value $\leq 0.05)$ with abdominal obesity were:
\end{abstract}


being married, not being white, having 8 or more years of schooling; having BMI indicative of overweight and generalized obesity and not consuming alcoholic beverages. Abdominal obesity, as well as generalized obesity, constitutes a major challenge to be addressed by health professionals, considering that a significant fraction of the population is in this circumstance. In this perspective, the prevention and control of abdominal obesity should provide actions that assist individuals in adopting a healthy lifestyle.

Keywords: Obesity; Body weight; Lifestyle, Chronic disease; Risk factors.

\section{Resumen}

La obesidad abdominal se asocia a un aumento exponencial del riesgo de morbimortalidad, ya que el patrón de distribución de la grasa corporal es el factor de riesgo más significativo en los procesos mórbidos que la obesidad generalizada. Estimar la prevalencia de obesidad abdominal e identificar los factores asociados. Estudio piloto, descriptivo, transversal con abordaje cuantitativo. La muestra de conveniencia estuvo formada por 50 individuos de 18 a 50 años. Inicialmente se realizó una valoración antropométrica de los participantes y luego se aplicó un cuestionario para definir el perfil y hábitos de la población con alteración de la circunferencia de cintura $(\geq 88 \mathrm{~cm}$ para mujeres $\mathrm{o} \geq$ $102 \mathrm{~cm}$ para hombres). Los datos se analizaron mediante estadística descriptiva y regresión lineal utilizando el software Epi Info ${ }^{\mathrm{TM}}$. Entre los participantes, el $58 \%(\mathrm{n}=29)$ tenía obesidad abdominal. Las variables que mostraron significancia ( $\mathrm{p}$-valor $\leq 0.05$ ) con la obesidad abdominal fueron: estar casado, no ser blanco, tener 8 o más años de escolaridad; tener un IMC indicativo de sobrepeso y obesidad generalizada y no consumir bebidas alcohólicas. La obesidad abdominal, así como la obesidad generalizada, constituye un gran desafío a ser abordado por los profesionales de la salud, considerando que una fracción importante de la población se encuentra en esta circunstancia. En esta perspectiva, la prevención y el control de la obesidad abdominal deben proporcionar acciones que ayuden a las personas a adoptar un estilo de vida saludable.

Palabras clave: Obesidad; Peso corporal; Estilo de vida, Enfermedad crónica; Factores de riesgo.

\section{Introdução}

A obesidade é uma doença crônica não transmissível (DCNT), descrita como acúmulo anormal ou excessivo de gordura corporal que apresenta repercussões negativas à saúde, como as doenças cardiovasculares (DCV), diabetes, distúrbios músculoesqueléticos e alguns tipos de cânceres (WHO, 2020). Refere-se a um problema de saúde pública e é considerada a mais significativa desordem nutricional no mundo, condicionada principalmente pelo perfil alimentar e de atividade física (WHO, 2000). Está associado a um aumento exponencial no risco de morbimortalidade, visto que o padrão de distribuição de gordura corporal é o fator de risco mais significativo em processos mórbidos do que a obesidade generalizada (Elffers et al., 2017).

Em 2016, mais de 650 milhões de indivíduos com 18 anos ou mais se enquadravam no quadro de obesidade, sendo referente a $13 \%$ da população mundial (WHO, 2020). Neste mesmo período, no Brasil, a taxa de prevalência de obesidade generalizada foi de 18,9\% na população adulta (Brasil, 2019). Dados de 2013 demonstraram que 52,1\% das mulheres possuíam uma circunferência da cintura maior ou igual a $88 \mathrm{~cm}$ e 21,8\% dos homens possuíam $102 \mathrm{~cm}$ ou mais, valores que determinam e estabelecem a obesidade abdominal em ambos os sexos (IBGE, 2013).

Considerando a importância da prevenção da obesidade, e mais especificamente da obesidade abdominal, a avaliação dos indivíduos deve abordar índice de massa corporal (IMC) em conjunto com medidas que refletem a adiposidade abdominal, como a circunferência da cintura (CC). Estas medidas comparadas a outros indicadores antropométricos são considerados mais adequados na previsão do risco de DCV na população geral, pois é um marcador que reflete indiretamente o risco cardiometabólico (Czernichow, Kengne, Stamatakis, Hamer \& Batty, 2011; Meller et al., 2014; Hsie et al., 2019).

Dados apresentados pela Organização Mundial da Saúde (OMS) apontam que as DCV são as principais causas de mortalidade mundial, tendo aproximadamente $31 \%$ dos registros de mortalidade no mundo (WHO, 2017), enquanto, no Brasil, são responsáveis por 27,7\% na taxa de óbito (Massa, Duarte \& Chiavegatto Filho, 2019), sendo a obesidade um fator de prédisposição para o surgimento e desenvolvimento dessas patologias (Carvalho et al., 2015). Atinente a isso, o acolhimento, o tratamento e o acompanhamento do indivíduo obeso, por vezes é realizado pela equipe da Atenção Primária, a qual é a principal porta de entrada e de comunicação entre os pontos da Rede de Atenção a Saúde (Brasil, 2014).

$\mathrm{Na}$ atenção primária a saúde, são atribuições inerentes ao enfermeiro, exercer atividades de promoção à saúde com a 
participação social, instituir a estratificação do risco para indivíduos obesos na unidade básica de saúde, bem como garantir o cuidado integral e multidisciplinar aos indivíduos que se caracterizam no quadro da obesidade, essencialmente em casos estabelecidos de comorbidades associadas (Brasil, 2017).

Diante do exposto e do cenário atual que remete a inúmeros desafios à assistência aos indivíduos obesos e a redução dos riscos as doenças cardiovasculares, definiu-se como objetivo do presente estudo estimar a prevalência de obesidade abdominal e identificar os fatores associados.

\section{Metodologia}

Trata-se de um estudo descritivo transversal, com abordagem quantitativa (Estrela, 2018). A amostra foi constituída por indivíduos de ambos os sexos entre 18 e 50 anos de um município do noroeste do Paraná.

A coleta de dados foi realizada no mês de abril de 2019 com uma amostra por conveniência de 50 indivíduos. A amostra foi selecionada da seguinte maneira: todos os indivíduos que adentraram na UBS foram abordados, questionados sobre os critérios de inclusão e exclusão do estudo e então os que atenderam a estes critérios foram convidados a participar da pesquisa. A coleta de dados foi finalizada quando 50 indivíduos responderam ao questionário. A UBS foi escolhida por conveniência, por se localizar próxima à Universidade. Os critérios de inclusão para o estudo foram: estar cadastrado na UBS, ter entre 18 e 50 anos e ser residente do Município local do estudo. Os critérios de exclusão incluíram: estar gestante no momento da pesquisa e puérperas, até 45 dias após o parto.

Os participantes foram submetidos à avaliação de medidas antropométricas de peso, estatura e circunferência de cintura (CC) e, posteriormente, a pesquisadora aplicou um questionário semi-estruturado com vistas a definir o perfil, hábitos e estilo de vida desta amostra.

Parâmetros técnicos para a verificação das medidas seguiram o manual de antropometria do Instituto Brasileiro de Geografia e Estatística (IBGE, 2013). Para a aferição do peso, os indivíduos foram orientados a permanecer em posição ortostática, posicionados sobre o centro da balança antropométrica digital para mensuração de peso e altura, com os pés descalços, trajando roupas leves. Para medida da estatura foi utilizada a régua antropométrica da balança, e os mesmos foram orientados a permanecer eretos e na posição ortostática, com os pés descalços e unidos.

A avaliação do IMC foi realizada pela divisão do peso corporal (em quilograma) pelo quadrado da altura (em metro). A classificação do IMC foi definida em obesidade, sobrepeso, peso normal e desnutrição de acordo com a Organização Mundial da Saúde (WHO, 2020).

Para a medida da CC foi orientado aos participantes manter a região da cintura livre de roupas e a permanecer em pé, descalço, com a blusa levantada, os braços flexionados e cruzados em frente ao tórax, pés afastados, abdômen relaxado e respirando normalmente. Foi utilizada uma fita métrica não flexível diretamente sobre a pele na região entre a última costela e a crista ilíaca, sendo a leitura feita no momento da expiração, tendo como parâmetro o valor de $\geq 88 \mathrm{~cm}$ para mulheres ou $\geq 102$ cm para homens (IDF, 2016).

Os dados coletados foram organizados em planilha do software Microsoft ${ }^{\circledR}$ Excel e analisados, por meio de estatística descritiva e regressão linear utilizando-se a versão 7 do software Epi Info ${ }^{\mathrm{TM}}$. Para a análise de correlação entre as variáveis foi utilizado a Regressão Linear, que constitui na aplicação de apenas uma variável explicativa para a determinação do comportamento esperado de Y (Silva, 2016).

Sendo assim, as variáveis com mais de uma categoria foram agrupadas da seguinte forma: idade 18 a 34 ou 35 a 50; raça e cor foram categorizados como branco ou não branco (pardos, amarelos e indígenas); escolaridade até 8 anos de estudo e 8 anos ou mais de estudo; renda familiar até 3 salários mínimos ou mais de 3 salários mínimos; tabagismo nunca fumou (não) ou fumante/ex-fumante (sim); consumo de bebida alcoólica nunca consumiu (não) ou socialmente/regularmente/quase 
sempre/todos os dias (sim); as variáveis consumo de frutas, legumes e verdura, carne vermelha, carne branca, doces, gorduras foram agrupadas da seguinte maneira: nunca, às vezes, regularmente/quase sempre/todos os dias. O comportamento sedentário foi classificado considerando o tempo por semana que o indivíduo apresenta comportamento sedentário sendo que até $7 \mathrm{~h} /$ semanais foi categorizado como não e acima 7h/semanais como sim (SBRN, 2017). O IMC foi categorizado da seguinte forma $<18$ a $<25$ (sem fator de risco) 25 a $<30$ (sobrepeso) e $>30$ (obeso) (WHO, 2020). A classificação de inatividade física foi estabelecida para os indivíduos com que apresentaram atividade física moderada a vigorosa menor que 150 minutos por semana, ou o mínimo de 75 minutos de atividade física vigorosa por semana (Brasil, 2019).

Esta pesquisa é um estudo piloto do projeto intitulado: "Eficácia de um programa multiprofissional na avaliação de fatores de risco cardiometabólico e tratamento da obesidade abdominal em dois municípios do noroeste do Paraná", liderado pela Universidade Estadual de Maringá em parceria com a Universidade Estadual do Paraná - Campus Paranavaí, financiado pela Fundação Araucária, com aprovação do comitê de ética em pesquisa da Universidade Estadual de Maringá, sob parecer ${ }^{\circ}$ 2.655.268. Todos os interessados que se enquadravam nos critérios de inclusão foram orientados sobre o estudo e consentiram sua participação por meio do Termo de Consentimento Livre e Esclarecido.

\section{Resultados}

Os participantes do estudo apresentaram uma média de idade de 35 anos. Sendo que $70 \%$ eram mulheres, destas, $62 \%$ eram casadas. Dos indivíduos com o estado civil, casadas, 70,9 \% apresentavam obesidade abdominal, com a idade superior a $30 \operatorname{anos}(54,8 \%)$.

A Tabela 1 apresenta as variáveis de sexo, raça/cor, escolaridade e renda familiar, que constituem as informações sociodemográficas dos indivíduos.

Tabela 1. Características sociodemográficas. Paranavaí (PR), Brasil, 2019.

\begin{tabular}{lccc}
\hline Variável & $\mathbf{n}$ & Obesidade abdominal (n) & $\begin{array}{c}\text { \% da variável entre os obesos } \\
\text { abdominais }\end{array}$ \\
\hline Sexo & 35 & 24 & $82,8 \%$ \\
Mulher & 15 & 5 & $17,2 \%$ \\
Homen & & & \\
Raça/cor & 20 & 12 & $41,4 \%$ \\
Branco & 30 & 17 & $58,6 \%$ \\
Não-branco & & 12 & $41,4 \%$ \\
Escolaridade & 14 & 17 & $58,6 \%$ \\
$<8$ anos de estudo & 36 & & \\
$>8$ anos de estudo & & 26 & $89,7 \%$ \\
Renda familiar & 43 & 3 & $10,3 \%$ \\
$\leq 3$ salários mínimos & 7 & & \\
$\geq 4$ salários mínimos & & & \\
\hline
\end{tabular}

Fonte: Elaborado pelos autores utilizando o software Microsoft@ Excel (2019).

A Tabela 2 demonstra a relação entre o Índice de Massa Corporal (IMC) e a quantidade de pessoas que foram classificadas com peso normal, sobrepeso e obesidade. 
Tabela 2. Relação entre IMC e obesidade abdominal. Paranavaí (PR), Brasil, 2019.

\begin{tabular}{lccc}
\hline & Variável & Obesidade abdominal (n) & $\begin{array}{c}\text { \% da variável entre os obesos } \\
\text { abdominais }\end{array}$ \\
\hline IMC & 16 & 2 & $6,9 \%$ \\
$18 \mathrm{a}<25$ & 17 & 10 & $34,5 \%$ \\
$25 \mathrm{a}<30$ & 17 & 17 & $58,6 \%$ \\
$\geq 30$ & & & \\
\hline
\end{tabular}

Intervalos do IMC: Peso Normal [18 a < 25], Sobrepeso [25 a < 30] e Obesidade [ $\geq 30]$.

Fonte: Elaborado pelos autores utilizando o software Microsoft@ Excel (2019).

Dentre os participantes com obesidade abdominal, 48,2\% relataram que apresentavam algum tipo de comorbidade, sendo que $31 \%$ eram hipertensos crônicos, $6,8 \%$ apresentavam diabetes mellitus, $6,8 \%$ hipotireoidismo e 3,4\% ansiedade.

A Tabela 3 exibe a relação entre a frequência do consumo de alimentos e o risco de desenvolver a obesidade entre os participantes.

Tabela 3. Consumo alimentar relacionado a obesidade abdominal. Paranavaí (PR), Brasil, 2019.

\begin{tabular}{|c|c|c|c|}
\hline Variável & $\mathbf{n}$ & Obesidade abdominal (n) & $\begin{array}{c}\% \text { da variável entre os obesos } \\
\text { abdominais }\end{array}$ \\
\hline \multicolumn{4}{|l|}{ Frutas } \\
\hline Nunca & 2 & 2 & $6,9 \%$ \\
\hline Às vezes & 23 & 13 & $44,8 \%$ \\
\hline Regularmente & 25 & 14 & $48,2 \%$ \\
\hline \multicolumn{4}{|c|}{ Legumes e verduras } \\
\hline Nunca & 0 & 0 & 0 \\
\hline Às vezes & 11 & 3 & $10,3 \%$ \\
\hline Regularmente & 39 & 26 & $89,7 \%$ \\
\hline \multicolumn{4}{|c|}{ Carne vermelha } \\
\hline Nunca & 0 & 0 & 0 \\
\hline Às vezes & 11 & 5 & $17,2 \%$ \\
\hline Regularmente & 39 & 24 & $82,8 \%$ \\
\hline \multicolumn{4}{|l|}{ Carne branca } \\
\hline Nunca & 2 & 1 & $3,4 \%$ \\
\hline Às vezes & 27 & 14 & $48,3 \%$ \\
\hline Regularmente & 21 & 14 & $48,3 \%$ \\
\hline \multicolumn{4}{|l|}{ Gordura } \\
\hline Nunca & 4 & 2 & $6,9 \%$ \\
\hline Às vezes & 21 & 10 & $34,5 \%$ \\
\hline Regularmente & 25 & 17 & $58,6 \%$ \\
\hline \multicolumn{4}{|l|}{ Doce } \\
\hline Nunca & 9 & 5 & $17,2 \%$ \\
\hline Às vezes & 27 & 16 & $55,2 \%$ \\
\hline Regularmente & 14 & 8 & $27,6 \%$ \\
\hline
\end{tabular}

Fonte: Elaborado pelos autores utilizando o software Microsoft® Excel (2019). 
A Tabela 4 mostra a relação entre a frequência dos hábitos de vida e o risco de desenvolver a obesidade entre os participantes.

Tabela 4. Relação entre a obesidade abdominal e hábitos de vida. Paranavaí (PR), Brasil, 2019.

\begin{tabular}{lccc} 
Variável & n & $\begin{array}{c}\text { Obesidade Abdominal } \\
\text { (n) }\end{array}$ & $\begin{array}{c}\text { \% da variável entre os obesos } \\
\text { abdominais }\end{array}$ \\
\hline Atividade física suficiente* & & & \\
Não & 29 & 12 & $41,4 \%$ \\
Sim & 21 & 17 & $86,6 \%$ \\
Tabagismo & & & \\
Nunca fumou & 44 & 25 & $86,2 \%$ \\
Fumante/ex-fumante & 6 & 4 & $13,8 \%$ \\
Consumo de álcool & & & $62,1 \%$ \\
Não & 27 & 18 & $37,9 \%$ \\
Sim & 23 & 11 & \\
\hline
\end{tabular}

*Atividade física suficiente: indivíduos que praticam pelo menos 150 minutos semanais de atividade física de intensidade moderada ou pelo menos 75 minutos semanais de atividade física de intensidade vigorosa.

Fonte: Elaborado pelos autores utilizando o software Microsoft@ Excel (2019).

Dentre o total da amostra, 50\% referiram considerar sua alimentação saudável, sendo 44,8\% obesos abdominais, enquanto 55,2\% dos obesos abdominais não consideravam a alimentação adequada.

Para verificar a existência de correlação significativa entre obesidade abdominal e as variáveis do estudo foi utilizado à análise estatística Regressão Linear, e o resultado desta análise é apresentado na Tabela 5. 
Tabela 5. Análise de correlação entre obesidade abdominal e as variáveis observadas. Paranavaí (PR), Brasil, 2019.

\begin{tabular}{lcc}
\hline Variável & Erro amostral & p-valor \\
\hline Cor/Raça & 0.12 & $0.01^{*}$ \\
Estado civil & 0.15 & $0.01^{*}$ \\
Idade & 0.15 & 0.23 \\
Sexo & 0.16 & 0.07 \\
Nível de escolaridade & 0.19 & $0.04^{*}$ \\
Renda familiar & 0.16 & 0.29 \\
IMC & 0.14 & $0.00^{*}$ \\
Consumo de bebida alcóolica & 0.15 & $0.05^{*}$ \\
Consumo de doce & 0.12 & 0.52 \\
Consumo de gordura & 0.11 & 0.33 \\
Consumo de legumes e verduras & 0.15 & 0.30 \\
Consumo de carne branca & 0.13 & 0.30 \\
Consumo de carne vermelha & 0.15 & 0.61 \\
Comorbidades & 0.13 & 0.90 \\
Atividade física insuficiente & 0.15 & 0.24 \\
Tabagismo & 0.17 & 0.27 \\
\hline
\end{tabular}

*p-valor significativo $\leq 0.05$.

* Regressão linear

Fonte: Elaborado pelos autores utilizando o software Microsoft® Excel (2019).

Dentre as variáveis analisadas, ser casado, não ser da raça branca; ter 8 anos ou mais de escolaridade; ter IMC indicativo de sobrepeso e obesidade e a variável da não consumo de bebida alcoólica têm correlação positiva (p-valor significativo) com a obesidade abdominal.

\section{Discussão}

A população analisada no estudo, em sua maioria, foi classificada com sobrepeso e obesidade, além disso, verificou-se uma alta prevalência de obesidade abdominal na população estudada, seguindo a tendência nacional (IBGE, 2013), também observada em um estudo realizado em São Francisco do Conde, na Bahia (Oliveira, West, Araújo, Brito \& Sobrinho, 2015).

A obesidade abdominal foi mais prevalente no sexo feminino. Essa heterogeneidade na distribuição entre os sexos corrobora com outros estudos realizados no Brasil (Oliveira, West, Araújo, Brito \& Sobrinho, 2015; Sabóia et al., 2016). Essa maior prevalência nos indivíduos do sexo feminino pode ser remetida à maior concentração de gordura corporal, devido às oscilações hormonais ocorridas no organismo da mulher no decorrer da vida (Barroso et al., 2017).

Indivíduos casados demonstraram significância positiva com a obesidade abdominal, podendo ser justificado pela associação com a idade mais desenvolvida do indivíduo neste estado civil, correspondendo ao transcurso do envelhecimento que promove modificações na taxa metabólica basal e no ritmo do metabolismo, fatores que propiciam o acúmulo de gordura corporal (Sabóia et al., 2016).

O padrão de distribuição de gordura corporal diverge entre as raças conforme os fatores correlacionados ao perfil socioeconômico, biológico e cultural (Eickemberg et al. 2020). Na amostra da pesquisa, não ser da raça branca apresentou significância estatística, sendo composta em sua maior parte pela raça parda, contrapondo-se parcialmente a literatura, que 
demonstra a prevalência de obesidade abdominal superior em homens brancos $(69,9 \%)$ e mulheres pardas $(63,3 \%)$, justificado pela menor concentração de gordura visceral nos descendentes de negros, quando comparados à raça branca (Eickemberg et al. 2020). Entretanto, homens e mulheres de descendência negra possuem pré-disposição para um maior acúmulo de tecido adiposo quando comparados aos brancos (Katzmarzyk et al. 2010), corroborando o resultado dessa pesquisa.

Possuir mais de oito anos de estudo impactou na elevada taxa de obesidade abdominal, assim como um estudo realizado em São Luís (Sabóia et al. 2016), que apresentou uma maior porcentagem de obesidade abdominal nos indivíduos com escolaridade a partir do ensino médio incompleto. Isso porque o nível de escolaridade influencia no poder aquisitivo, o que por sua vez facilita o acesso aos industrializados e de rápido preparo.

Todavia, os resultados contradizem a literatura nacional em respeito a DCNT, indivíduos com baixa escolaridade vem sendo associados a um maior predomínio de obesidade, bem como doenças relacionadas.

Por outro lado, a maior prevalência de obesidade abdominal por renda familiar, foi encontrada em participantes com baixo nível socioeconômico. Usualmente, esses indivíduos evidenciam maior ingesta de alimentos de menor valor nutricional, ricos em gorduras e açúcares, quando comparados com alimentos de origem proteica. Este pode ser o coeficiente de influência no índice de obesidade abdominal nos indivíduos com baixo nível socioeconômico (Oliveira, West, Araújo, Brito \& Sobrinho, 2015).

A prevalência de obesidade abdominal tem aumentado quando comparado aos anos precedentes e é superior em relação à prevalência de obesidade generalizada (Barroso et al. 2017). Isso pode ser observado neste estudo, que demonstrou uma prevalência de obesidade generalizada de 34\%, instituída através do cálculo de IMC, enquanto a prevalência de obesidade abdominal foi de 58\%. Essa variável corrobora com um estudo realizado em um município do Rio de Janeiro, no qual foi estabelecida uma prevalência de 70\% de obesidade generalizada e 95\% de obesidade abdominal (Barroso et al. 2017).

Contudo, a correlação entre a obesidade abdominal estabelecida através da CC e IMC referente ao sobrepeso e obesidade possuem um p-valor de relevância, sendo o mais significativo deste estudo. Um estudo elaborado na Ásia constatou que os parâmetros antropométricos IMC e CC estão associados a fatores de risco para síndrome metabólica e DCV (Chuang et al., 2012). Outra pesquisa realizada no Sul do Brasil investigou a relação entre o IMC e CC e demonstrou que a combinação entre esses indicadores é um método para identificar indivíduos com aumento do risco para desenvolver doença cardiovascular (Turuchima, Ferreira \& Bennemann RM, 2015).

As DCNT como a hipertensão arterial sistêmica, diabetes mellitus e dislipidemias, acrescidos à obesidade abdominal, são condições relevantes, em virtude a coligação com o risco de geração de doenças cardiovasculares, problemas metabólicos e dentre outros agravos à saúde (Barroso, 2017), sendo um fator de alerta para o presente estudo, uma vez que a comorbidade mais prevalente entre os participantes com obesidade abdominal foi à hipertensão arterial sistêmica, com 31\% entre os obesos abdominais.

A alimentação saudável é reconhecida como um componente fundamental para a promoção da saúde e prevenção de DCNT (IFPRI, 2014). O Ministério da Saúde criou um Guia Alimentar Saudável para a população, com o intuito de trabalhar com a promoção da saúde no aspecto que tange a obesidade e DCNT (Brasil, 2014). Nesse sentido, destaca-se a necessidade dos profissionais utilizarem estas ferramentas no planejamento das ações práticas que possuem como foco a prevenção e a promoção.

A análise de correlação entre a obesidade abdominal e as variáveis do hábito de alimentação dos participantes não apresentou p-valor significativo, contudo, é possível observar que a porcentagem de maior prevalência entre os obesos abdominais concentrou-se no maior consumo de legumes/verduras, carnes vermelhas e gorduras. A relação entre a obesidade abdominal e o maior consumo de legumes e verduras não é descrito na literatura, já o consumo de carne vermelha, além de estar vinculada ao aparecimento de diabete mellitus e resistência insulínica, esteve intimamente associado ao aumento da circunferência da cintura em um estudo de realizado no ano de 2019 com 15.105 funcionários públicos de instituições de pesquisa 
(Aprelini et al., 2019). Já o consumo demasiado de gordura saturada está relacionado ao desenvolvimento de DCNT (Brasil, 2017).

Mais da metade da amostra da população com obesidade abdominal referiu realizar atividade física insuficiente. Dentre os comportamentos e hábitos saudáveis a prática de atividade física propicia benefícios além do controle da obesidade, e constitui um importante fator da prevenção de DCV (Brasil, 2017). Ações para a estimulação destas praticas por parte da APS incluem as academias da saúde, recintos voltados para a replicação de promoção a saúde, espaços propícios para a captação de indivíduos com DCNT (WHO, 2020)

O (n) amostral demonstrou associação estatística com a variável da não ingestão de bebida alcoólica, divergindo da literatura, que aponta o tabagismo e a ingestão de álcool como coeficientes conexão com a obesidade abdominal, também responsável pela maioria dos óbitos por doenças e agravos não transmissíveis (Sabóia et al. 2016).

A obesidade abdominal constitui-se como um grande desafio a ser trabalhado pelos profissionais da saúde, gestores e formuladores de políticas públicas, considerando que uma fração significativa da população se encontra nessa circunstância (Aprelini et al., 2019). Nessa perspectiva, a prevenção e o controle da obesidade abdominal devem prover ações que auxiliem os indivíduos na adoção de estilo de vida saudável (Brasil, 2014).

A promoção e prevenção da obesidade tem como preceptor substancial para esta ação o profissional enfermeiro, o qual desenvolve ações que tencionam a melhoria da qualidade de vida, sucedendo através da consulta de enfermagem, o qual abrange a monitorização dos dados antropométricos, aconselhamento aos indivíduos de todas as faixas etárias, incluindo aspectos profiláticos e de remediação, orientações sobre a importância de hábitos alimentares saudáveis, bem como a relevância da necessidade da realização de atividades físicas (Braga et al. 2017).

Por se tratar de um projeto piloto, o estudo apresenta como limitação uma amostra pequena proveniente de apenas uma Unidade Básica de Saúde do município, porém esta amostra permitiu realizar uma análise estatística que demonstrou associações entre as variáveis estudadas e a obesidade abdominal.

\section{Considerações Finais}

A maioria dos participantes do estudo apresentou obesidade abdominal, sendo mais prevalente em indivíduos com mais idade e no sexo feminino. As variáveis que apresentaram significância positiva com a obesidade abdominal no estudo foram: ser casado; não ser da raça branca; ter 8 anos ou mais de escolaridade; ter IMC indicativo de sobrepeso e obesidade generalizada; não ingestão de bebida alcóolica. Este estudo tem o intuito de incentivar o planejamento de atividades de educação permanente em saúde para melhorar o atendimento prestado a esta população nos serviços públicos de saúde, além de servir como apoio para outros estudos.

O conhecimento dos fatores de risco associados ao desenvolvimento da obesidade abdominal podem subsidiar os serviços de saúde na promoção de ações educativas para estimular um estilo de vida saudável com a adesão de uma dieta equilibrada e prática de atividades físicas regulares. Alinhado a essa perspectiva, é imprescindível o investimento nessa temática, visto que a capacitação das equipes de saúde asseguram o cuidado integral para conscientizar o usuário a assumir o papel de protagonista durante a recuperação. Sugere-se portanto, estudos futuros que aprofundem o olhar sobre os fatores de risco para a obesidade e sua relação com características sociodemográficas, para aprimoramento das políticas públicas de saúde com foco na prevenção e tratamento da obesidade abdominal.

\section{Referências}

Aprelini, C. M. O., Luft, V. C., Meléndez, G. V., Schmidt, M. I., Mill, J. G., Molina, M. D. C. B. (2019). Consumo de carne vermelha e processada, resistência insulínica e diabetes no Estudo Longitudinal de Saúde do Adulto (ELSA-Brasil). Revista Panamericana de Salud Pública, 43: e40. https://doi.org/10.26633\%2FRPSP.2019.40 
Barroso, T. A., Marins, L. B., Alves, R., Gonçalves, A. C. S., Barroso, S. G. \& Rocha, G. S. (2017). Associação entre a obesidade central e a incidência de doenças e fatores de risco cardiovascular. International Journal of Cardiovascular Sciences, 30(5): 416-424 http://doi.org/10.5935/2359-4802.20170073

Braga, V. A. S., Jesus, M. C. P., Conz, C. A., Tavares, R. E., Silva, M. H. \& Merighi, M. A. B. (2017). Intervenções do enfermeiro às pessoas com obesidade na Atenção Primária à Saúde: revisão integrativa. Revista da Escola de Enfermagem da USP, 51:e03293. https://doi.org/10.1590/s1980-220x2017019203293

Brasil. (2014). Ministério da Saúde. Secretaria de Atenção à Saúde. Departamento de Atenção Básica. Estratégias para o cuidado da pessoa com doença crônica: obesidade. $1^{\text {a }}$ ed. Brasília: Ministério da Saúde. http://bvsms.saude.gov.br/bvs/publicacoes/estrategias_cuidado_doenca_cronica_obesidade_cab38.pdf

Brasil. (2017). Agência Nacional de Saúde Suplementar. Diretoria de Normas e Habilitação dos Produtos. Gerência-Geral de Regulação Assistencial. Gerência de Monitoramento Assistencial. Coordenadoria de Informações Assistenciais. Manual de diretrizes para o enfrentamento da obesidade na saúde suplementar brasileira. Rio de Janeiro: Agência Nacional de Saúde https://www.ans.gov.br/images/Manual_de_Diretrizes_para_o_Enfrentamento_da_Obesidade_na_Sa\%C3\%BAde_Suplementar_Brasileira.pdf

Brasil. (2017). Ministério da Saúde. Portaria $n^{o}$ 2.436/2017. Aprova a Política Nacional de Atenção Básica, estabelecendo a revisão de diretrizes para a organização da Atenção Básica, no âmbito do Sistema Único de Saúde (SUS). Brasília: Ministério da Saúde. https://bvsms.saude.gov.br/bvs/saudelegis/gm/2017/prt2436_22_09_2017.html

Brasil. (2019). Ministério da Saúde. Secretaria de Vigilância em Saúde. Departamento de Análise em Saúde e Vigilância de Doenças Não Transmissíveis. VIGITEL BRASIL 2019: vigilância de fatores de risco e proteção para doenças crônicas por inquérito telefônico. Ministério da Saúde. Recuperado de: https://portalarquivos.saude.gov.br/images/pdf/2020/April/27/vigitel-brasil-2019-vigilancia-fatores-risco.pdf

Carvalho, C. A., Fonseca, P. C. A., Barbosa, J. B., Machado, S.P., Santos, A.M. \& Silva, A. A. M. (2015). Associação entre fatores de risco cardiovascular e indicadores antropométricos de obesidade em universitários de São Luís, Maranhão, Brasil. Ciência \& Saúde Coletiva, 20(2): 479-490. https://doi.org/10.1590/1413-81232015202.02342014

Chuang, H. H., Li, W. C., Sheu, B. F., Liao, S. C., Chen, J. Y., Chang, K. C. \& Tsai, Y. W. (2012). Correlation between body composition and risk factors for cardiovascular disease and metabolic syndrome. Biofactors, 38(4): 284-291. https://doi.org/10.1002/biof.1027

Czernichow, S., Kengne, A. P., Stamatakis, E., Hamer, M. \& Batty, G. D. (2011). Body mass index, waist circumference and waist-hip ratio: which is the better discriminator of cardiovascular disease mortality risk? Evidence from an individual-participant meta-analysis of 82.864 participants from nine cohort studies. Obesity Reviews, 12(9): 680-687. https://doi.org/10.1111/j.1467-789x.2011.00879.x

Eickemberg, M., Amorim, L. D. A. F., Almeida, M. C. C., Pitanga, F. J. G., Aquino, E. M. L., Fonseca, M. J. M. \& Matos, S. M. A. (2020). Obesidade abdominal no ELSA-Brasil: construção de padrão-ouro latente e avaliação da acurácia de indicadores diagnósticos. Ciência \& Saúde Coletiva, 25(8): 2985-2998. https://doi.org/10.1590/1413-81232020258.20992018

Elffers, T. W., Mutsert, R., Lamb, HJ, Roos, A., Dijk, K. W. V., Rosendaal, F. R., Jukema, J. W. \& Trompet, S. (2017). Body fat distribution, in particular visceral fat, is associated with cardiometabolic risk factors in obese women. PLOS ONE, 12(9), e0185403. https://doi.org/10.1371/journal.pone.0185403

Estrela, C. (2018). Metodologia Científica: Ciência, Ensino, Pesquisa. (3a ed.), Artes Médicas.

Hsie, Y. H., Wu, M. F., Yang, P. Y., Liao, W. C., Hsieh, Y. H., Chang, Y. J. \& Lin, I. C. (2019). What is the impact of metabolic syndrome and its components on reflux esophagitis? A cross-sectional study. BMC Gastroenterology, 19(1): e33. https://doi.org/10.1186/s12876-019-0950-z

Instituto Brasileiro de Geografia e Estatística - IBGE. (2010). Censo demográfico: resultados preliminares do município de Paranavaí, PR. Brasília: Instituto Brasileiro de Geografia e Estatística. Recuperado de: https://cidades.ibge.gov.br/brasil/pr/paranavai/panorama

Instituto Brasileiro de Geografia e Estatística - IBGE. (2013). Ministério do Planejamento, Orçamento e Gestão. Pesquisa Nacional de Saúde 2013: Manual de Antropometria. Rio de Janeiro: Instituto Brasileiro de Geografia https://www.pns.icict.fiocruz.br/arquivos/Novos/Manual\%20de\%20Antropometria\%20PDF.pdf

Instituto Brasileiro de Geografia e Estatística - IBGE. (2013). Pesquisa Nacional de Saúde (PNS) 2013. Brasília: Instituto Brasileiro de Geografia e Estatística.:https://www.pns.icict.fiocruz.br/ 5

International Diabetes Federation - IDF. (2006). The IDF consensus worldwide definition of the metabolic syndrome. Brussels: International Diabetes Federation. Recuperado de: https://www.idf.org/e-library/consensus-statements/60-idfconsensus-worldwide-definitionof-the-metabolic-syndrome.html

International Food Policy Research Institute - IFPRI. (2014). Global nutrition report 2014: actions and accountability to accelerate the world's progress on nutrition. Washington: International Food Policy Research Institute. https://ebrary.ifpri.org/digital/collection/p15738coll2/id/128484

Katzmarzyk, P. T., Bray, G. A., Greenway, F. L., Johnson, W. D., Newton Junior, R. L., Ravussin, E., Ryan, D. H., Smith, S. R. \& Bouchard, C. (2010). Racial differences in abdominal depot-specific adiposity in white and African American adults. The American Journal of Clinical Nutrition, 91(1): 7-15. https://doi.org/10.3945/ajen.2009.28136

Massa, K. H. C., Duarte, Y. A. O. \& Chiavegatto Filho, A. D. P. (2019). Análise da prevalência de doenças cardiovasculares e fatores associados em idosos, 2000-2010. Ciência \& Saúde Coletiva, 24(1): 105-114. http:// doi.org/10.1590/1413-81232018241.02072017

Meller, F. O., Ciochetto, C. R., Santos, L. P., Duval, P. A., Vieira, M. F. A. \& Schäfer, A. A. (2014). Associação entre circunferência da cintura e índice de massa corporal de mulheres brasileiras: PNDS 2006. Ciência \& Saúde Coletiva, 19(1): 75-82. https://doi.org/10.1590/1413-81232014191.2000

Oliveira, L. C., West, L. E. M., Araújo, E. A., Brito, J. S. \& Sobrinho, C. L. N. (2015). Prevalência de adiposidade abdominal em adultos de São Francisco do Conde, Bahia, Brasil, 2010. Epidemiologia \& Serviços de Saúde, 24(1): 135-144. https://doi.org/10.5123/S1679-49742015000100015

Sabóia, R. S., Araújo, A. P., Barbosa, J. M. A., Galvão, C. E. P., Cruvel, J. M. S. \& Ferreira, S. C. N. (2016). Obesidade abdominal e fatores associados em adultos atendidos em uma clínica escola. Revista Brasileira em Promoção da Saúde, 29(2): 259-267. https://doi.org/10.5020/18061230.2016.p259 
Research, Society and Development, v. 10, n. 6, e45110615708, 2021

(CC BY 4.0) | ISSN 2525-3409 | DOI: http://dx.doi.org/10.33448/rsd-v10i6.15708

Sedentary Behavior Research Network - SBRN. (2017). Final definitions, caveats and examples of key terms from the Sedentary Behavior Research Network Terminology Consensus Project. Ontario: Sedentary Behavior Research Network. https://www.sedentarybehaviour.org/sbrn-terminology-consensusproject/portuguese-brazil-translation/

Silva, J. P. B. C. (2016). Modelos de regressão linear e logística utilizando o software R. Dissertação [Mestrado em Estatística, Matemática e Computação]. Lisboa: Universidade Aberta; 2016. https://repositorioaberto.uab.pt/handle/10400.2/6129

Turuchima, M. T., Ferreira, T. N. \& Bennemann, R. M. (2015). Associação entre indicadores antropométricos (IMC e CC) em relação ao risco para doenças cardiovasculares. Saúde e Pesquisa, 8: 55-63. https://periodicos.unicesumar.edu.br/index.php/saudpesq/article/view/3847

World Health Organization - WHO. (2000). Obesity: preventing and managing the global epidemic. WHO Technical Report Series 894. World Health Organization. https://www.who.int/nutrition/publications/obesity/WHO_TRS_894/en/

World Health Organization - WHO. (2017). Cardiovascular diseases (CVDs). World Health Organization. https://www.who.int/es/news-room/factsheets/detail/cardiovascular-diseases-(cvds)

World Health Organization - WHO. (2020). Obesity and overweight. World Health Organization (Who); 2020. https://www.who.int/en/news-room/factsheets/detail/obesity-and-overweight 